\title{
Improving Head Orientation Prediction with Electromyography Signals in a Virtual Reality Flight Simulator
}

\author{
Dongsu $\mathrm{Wu}^{1,}$ a , Peng $\mathrm{Li}^{2}$, Lin Wang ${ }^{1}$, Lihao Liu ${ }^{1}$ and Hongbin $\mathrm{Gu}^{1}$ \\ ${ }^{1}$ College of Civil Aviation, Research Center for Flight Simulation and Advanced Training Engineering \\ Technology, Nanjing University of Aeronautics and Astronautics, Nanjing 211106,China \\ ${ }^{2}$ College of Automobile and Traffic Engineering, Nanjing Forestry University, Nanjing 210037, China \\ atissle@nuaa.edu.cn
}

Keywords: head tracking, electromyography, virtual reality, time-delay compensation, Kalman filter.

\begin{abstract}
As the control input of the virtual reality flight simulator, precise head tracking with no time-delay is essential for user immersion and training effect. We present the results of applying a modified Kalman filter to improve head orientation prediction. A piecewise constant acceleration approach with an electromyography based input estimation is suggested. Head angular acceleration is estimated by a trained feed-forward neural network and fed into the modified Kalman filter. Experimental results show that the proposed method tracks head motion precisely under both smooth and abrupt motion conditions.
\end{abstract}

\section{Introduction}

Full flight simulators(FFS) brings high-level fidelity for initial and recurrent pilot training and is widely used by major airlines. Due to high purchase, deployment and usage cost of FFS, nowadays airlines are seeking more affordable flight training devices to meet increasing training demands[1]. With the help of emerging virtual reality(VR) technology, flight simulator can be easily built with cheap COTS parts and flexibly reconfigured to different types of aircrafts[2]. Cockpit environment is constructed with 3D computer graphics instead of real parts, displayed in the helmet mounted display(HMD), and changed real-time with the pilot's head motion[3]. However, the fidelity of VR flight simulator extremely depends on the head tracking performance, the perceived time-delay between head motion and display will cause a loss of immersion and even serious simulation sickness for the user.

An effective method for reducing time-delay is to predict the future orientation of the head. Predictive filtering including Extended Kalman filter(EKF), unscented Kalman filter(UKF) and particle filter(PF) is widely used for compensation of time-delay. Although UKF and PF are more accurate for nonlinear and non-Gaussian system, Rhijn and etc.[4] found EKF have similar performance with UKF and PF in head orientation prediction and filtering. Furthermore, EKF has less equations and thus can reduce computation delay. Since head motion is random and unpredictable, the regular head model assumes constant velocity (CV) or constant acceleration (CA) property between two sensor samples[5]. That works fine for smooth head movement and introduces obvious performance degradation under abrupt head movement conditions[6]. A completely different approach to predict human motion is to use physiological signals such as electromyography(EMG)[7]. Neck muscles will generate myoelectric signals before muscle contraction and can be used as an auxiliary reference signal to improve the head orientation prediction. Baniv and etc.[8] trained a recurrent neural network with seven EMG features and estimated predictive head velocity. Since types and ranges of head motions are various between different subjects, collecting enough data and training a generalized neural network is a hard task. If the motion type is much different from the trained ones, the overall tracking performance will degraded rapidly.

In this paper, we propose a new framework for predicting head orientation prediction using a Kalman filter with CA model to track the slow motion and an EMG based angular acceleration estimator to compensate the onset predictor error. Experimental results show that the proposed 
method ensures the predictive tracking performance on both smooth and abrupt head motion conditions.

\section{Modified Kalman Filter based Head Orientation Prediction with CA model and Input Estimation}

Head rotation is a dynamic nonlinear process and has strong stochastic characteristics. We may focus on some object for a long time, and then suddenly turn our heads to look at another object. The dynamic process of head rotation is very similar to that of the maneuvering target problem, so a popular approach is to model the head kinematics with piecewise approximation model such as CA model. The head is assumed to move at a constant acceleration during the sampling interval and driven by white noise in a random walk model. The dynamic equations of CA model can be represented as

$$
\begin{aligned}
& X(k)=F(k \mid k-1) X(k-1)+G(k-1) w(k-1) \\
& Z(k)=H(k) X(k)+v(k) \\
& \text { Where } X(k)=\left[\begin{array}{c}
x(k) \\
\dot{x}(k) \\
\ddot{x}(k)
\end{array}\right], F(k \mid k-1)=\left[\begin{array}{ccc}
1 & T & T^{2} / 2 \\
0 & 1 & T \\
0 & 0 & 1
\end{array}\right], G(k)=\left[\begin{array}{c}
T^{3} / 6 \\
T^{2} / 2 \\
T
\end{array}\right], H(k)=\left[\begin{array}{lll}
0 & 1 & 0
\end{array}\right], T \text { is }
\end{aligned}
$$

the sampling interval; The process noise $w(k)$ is Gassian white noise , $w(k) \sim(0, Q(k))$ and $E\left[w(k) w^{T}(j)\right]=Q(k) \delta_{k-j}$ ( $\delta_{k-j}$ is Kronecker delta function); The measurement noise $v(k)$ is Gassian white noise, $v(k) \sim(0, R(k))$ and $E\left[v(k) v^{T}(j)\right]=R(k) \delta_{k-j}, \delta_{k-j}=\left\{\begin{array}{ll}1 & \text { if } i=j \\ 0 & \text { if } i \neq j\end{array}\right.$.

The motion tracking sensor is an inertial sensor with three angular velocities output, corresponding yaw, roll and pitch coordinates. The selection of the measurement noise is based on the inertial sensor noise level, whilst process noise depends on the variance of the tracking target and is chose by trail and error to get good tracking performance.

Kalman filter is a sequential data processing method, which estimated new state by recursively updating and is simple to implement. Furthermore, Kalman filter, explicitly considering statistical characteristics of estimated state variables and measured signals, is suitable to predict head motion with random disturbances. The basic prediction equations of Kalman filter can be represent as

States Prediction Equation:

$\hat{X}(k \mid k-1)=F(k \mid k-1) \hat{X}(k-1)$

Variances Prediction Equation:

$$
P(k \mid k-1)=F(k \mid k-1) P(k-1 \mid k-1) F^{T}(k \mid k-1)+G(k-1) Q(k-1) G^{T}(k-1)
$$

Kalman Gain Equation:

$$
K(k)=P(k \mid k-1) H^{T}(k)\left[H(k) P(k \mid k-1) H^{T}(k)+R(k)\right]^{-1}
$$

Innovation Update Equation:

$$
\hat{X}(k \mid k)=\hat{X}(k \mid k-1)+K(k)[Z(k)-H(k) \hat{X}(k \mid k-1)]
$$

Variances Update Equation:

$$
P(k \mid k)=[I-K(k) H(k)] P(k \mid k-1)
$$

Since the CA model assumes a piecewise constant acceleration process between samples. When head motion is abrupt, this assumption will be invalid and thus introduce large prediction error. Kiruluta and etc. [6] suggested add an input term to the acceleration process. The term is like a step discontinuity in acceleration between sampling interval to represent the sudden head motion. The modified acceleration process becomes

$$
\ddot{x}(k)=\ddot{x}(k-1)+u(k)+T w(k-1)
$$


Where $u(k)$ is the additional acceleration bias term. Gutman and Velger[9] estimated $u(k)$ with a running window curve fitting algorithm. However, the estimation must consider the trade-off between maneuver detection time and input estimation accuracy. Since the electromyography signals contains angular acceleration information before the head actually moves, we consider using electromyography to estimate the additional acceleration bias term $u(k)$.

\section{Electromyography Based Angular Acceleration Estimation}

EMG signal of human neck is the combination effect of about 30 muscles. To simplify the system deployment, we wish use as few electrodes as possible. After make experiments in different locations[10], we chose to put the electrodes on the belly of Sternocleidomastoid (SCM) where electromyography signal strength is monotonically related to the head motion. The Electromyography signal data was collected at $1200 \mathrm{~Hz}$ and the inertial sensor data was $120 \mathrm{~Hz}$ as a reference signal. The subjects were asked to turn head horizontally from left to right turn and backwards.

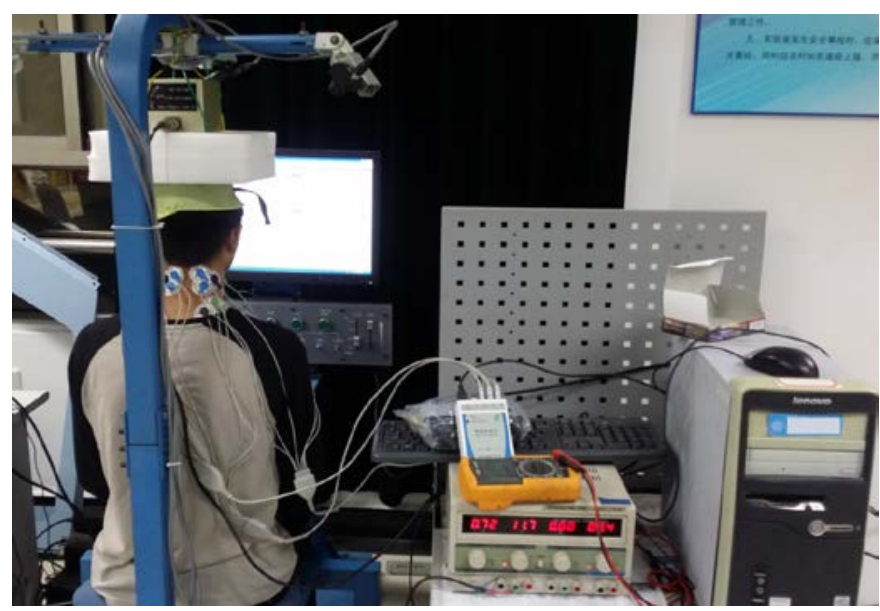

Fig. 1 Experiment Platform

Using EMG to estimate angular acceleration is actually a nonlinear mapping problem, which can be solved by pattern recognition(PR) techniques such as neural networks. The inputs of PR methods are features which are extracted from raw signals and best represented the system characteristics. Both amplitude and frequency of EMG signal carry head motion information, so we choose five features from both time and frequency domain of EMG signal(Table 1). The features are derived from the raw EMG signal over a 24ms moving window.

Table 1 EMG Features

\begin{tabular}{|c|c|}
\hline Feature & Description \\
\hline Mean Absolute Value & Mean absolute angular acceleration within a window \\
\hline Number of Zero-Crossing & $\begin{array}{c}\text { Number of points where the angular acceleration was } \\
\text { zero within a window }\end{array}$ \\
\hline Average Frequency & Instantaneous frequency at each time-step \\
\hline Waveform Length & Sum of waveform within a window \\
\hline Slope Sign Change & $\begin{array}{c}\text { Slope Sign Change of Angular acceleration within a } \\
\text { window }\end{array}$ \\
\hline
\end{tabular}

A feed-forward neural network with 50 hidden neurons was used and training algorithm is Levenberg-Marquardt. Since EMG signals precede actual motion about 30-70ms and the actual anticipating time is hard to measure, we suppose the EMG signals contain combined information of this time span and shift inertial angular velocities forward 50ms, then differentiate and smooth them to be angular acceleration signals as the training reference data for neural network. 


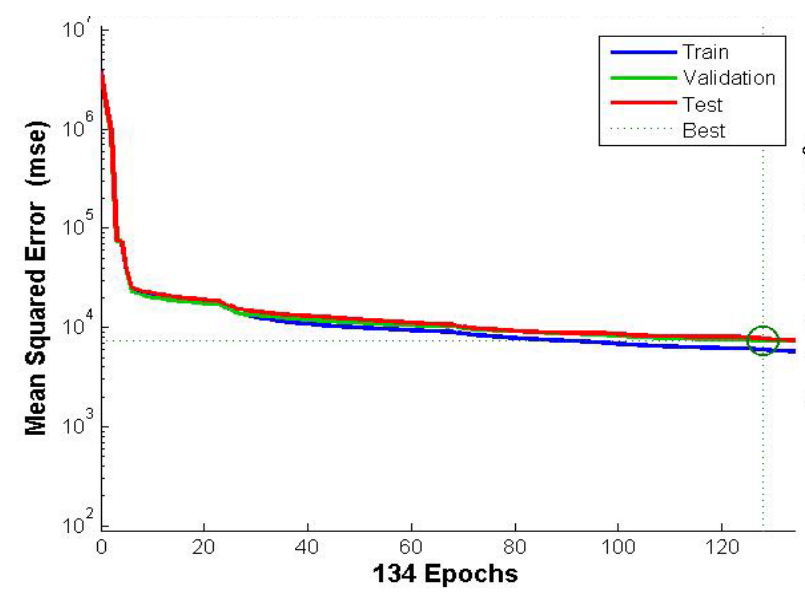

Fig. 2 NN Training Curve

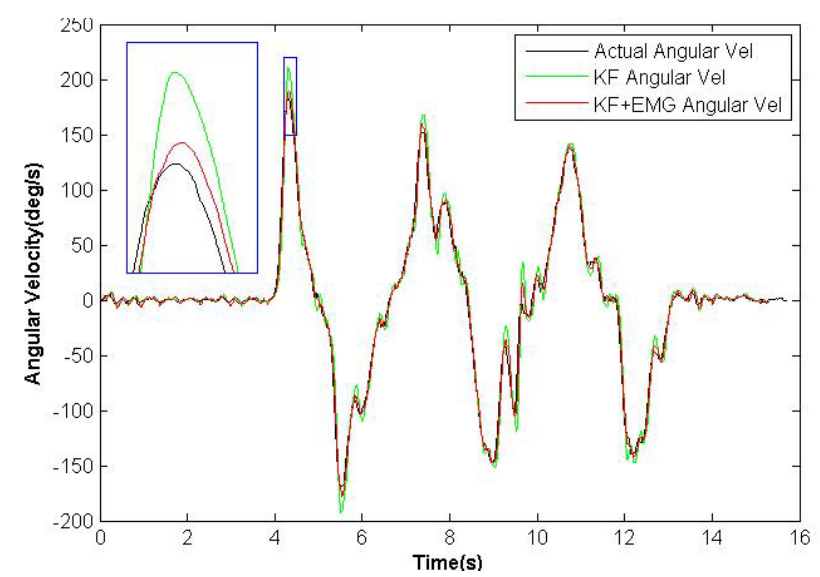

Fig. 4 Comparison of Angular Acceleration

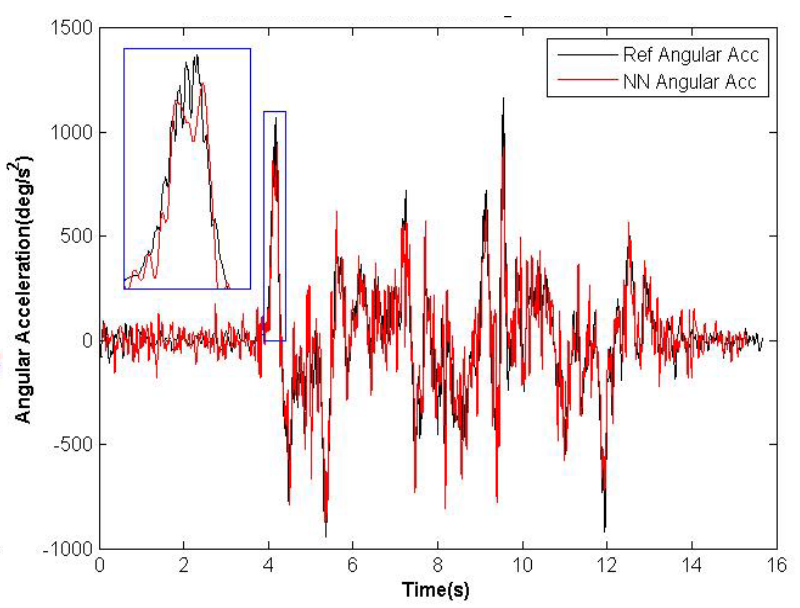

Fig. 3 Comparison of Angular Acceleration

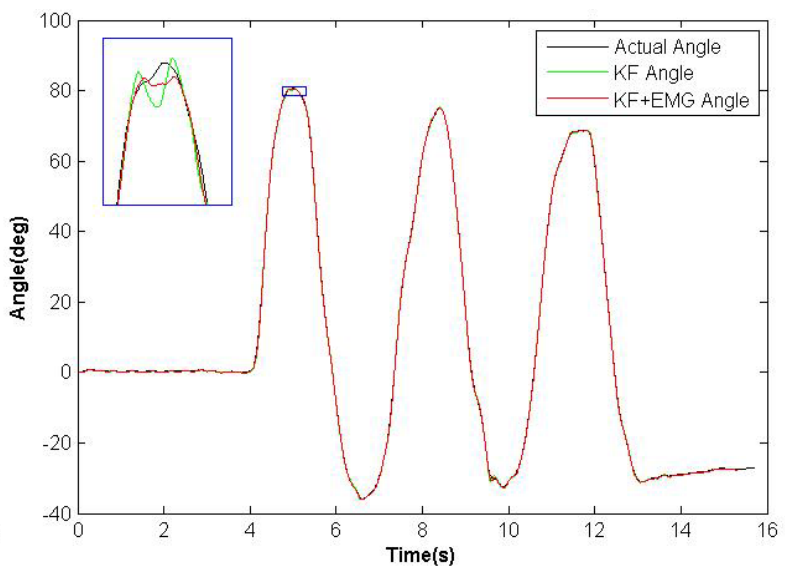

Fig. 5 Comparison of Azimuth Angle

Fig.2 presents qualitative training results for neural network. The angular acceleration of head horizontal rotation can be successfully mapping from EMG features through neural network. The total training process was very fast and ended after about 128 epochs. The estimated angular acceleration curve from feed-forward neural network is coincide with the actual acceleration which we got from the inertial sensor, differentiated and shifted forward 50ms. Fig. 4 and 5 compare the prediction performance of KF and modified KF with acceleration estimation methods. Both methods perform well during slow and smooth motion, while in the period of abrupt motion, the modified KF yields better performance.

Table 2 Comparison of ME and RMSE

\begin{tabular}{|c|c|c|c|c|}
\hline Method & ME & RMSE & ME(window) & RMSE(window) \\
\hline$K F$ & $0.0066^{\circ} / \mathrm{s}$ & $9.7156^{\circ} / \mathrm{s}$ & $13.4572^{\circ} / \mathrm{s}$ & $19.7447^{\circ} / \mathrm{s}$ \\
\hline$K F+E M G$ & $0.0063 \%$ & $5.2242^{\circ} / \mathrm{s}$ & $3.8477^{\circ} / \mathrm{s}$ & $8.4255^{\circ} / \mathrm{s}$ \\
\hline
\end{tabular}

Table 2 compares mean errors(ME) and root mean square errors(RMSE) of angular velocities between the sensor and tracking filters, on both overall and small windows of abrupt motion condition. The ME and RMSE results coincide with the conclusion we drew from above. We know that ME represents the trend of the mean value drift and RMSE is similar with the variance. On the slow motion condition, both methods has almost the same prediction ability. However, during the period of rapid head motion, modified Kalman filter we proposed predicts the raw velocity more accurately and overall ME and RMSE drops over 60\% compared with conventional KF. Actually, in the proposed method, we use the anticipated "real" head acceleration information instead of a "predicted" extrapolation. 


\section{Summary}

In this work, the effect of applying a modified Kalman filter with EMG based head angular acceleration estimation to predict both smooth and abrupt head dynamics in virtual reality is studied. The traditional Kalman filter with CA model can track head motion precisely under smooth and slow head movement. However, it performs not well when head rotate abruptly. Since EMG signals contain anticipated kinematics information, we try to reconstruct angular acceleration from EMG with the help of neural network, and then use it as an input estimation into the Kalman filter. Experimental results show the proposed method is superior to the traditional one.

\section{Acknowledgements}

This work is financially supported by National Natural Science Foundation of China (No.51205195, No.61039002), the Natural Science Foundation of Jiangsu Province(BK20130981), the Fundamental Research Funds for the Central Universities (No.NS2015064).

\section{References}

[1] L. Thomas, B. Edward and B. C. Judith, Initiative towards more affordable flight simulators for U.S. commuter airline training, Proc. Royal Aeronautical Society Conf. on Training, Lowering the Cost, Maintaining the Fidelity (1996), p. 2.1-2.17.

[2] T. Aslandere, D. Dreyer, F. Pankratz, R. Schubotz. A Generic Virtual Reality Flight Simulator. Virtuelle und Erweiterte Realitt, 11. Workshop der GI-Fachgruppe VR/AR, Shaker Verlag, Aachen, (2014), p.1-13.

[3] Hongbin Gu, Dongsu Wu, Hui Liu. Development of a Novel Low-Cost Flight Simulator for Pilot Training. International Conference on Control, Automation, Robotics and Vision, Bangkok, (2009), p.680-684.

[4] A. V. Rhijn, R. V. Liere, and J. D. Mulder, An analysis of orientation prediction and filtering methods for VR/AR, in Proc. IEEE Virtual Reality Conf. (2005), p. 67-74.

[5] Ma Deng-wu, Fan Geng. Predictive Algorithm Simulation of Head Motion in VR System Based on IMM. Journal of System Simulation. Vol.21, No.24 (2009), p.7817-7820.

[6] A. Kiruluta, M. Eizenman, and S. Pasupathy. Predictive Head Movement Tracking Using a Kalman Filter. IEEE Trans. On Sytems, Man, and Cybernetics-Part B: Cybernetics, Vol.27, No.2 (1997), p.326-331.

[7] Matthew R. Williams, Robert F. Kirsch. Evaluation of Head Orientation and Neck Muscle EMG Signals as Command Inputs to a Human-Computer Interface for Individuals with High Tetraplegia, IEEE Trans. On Neural Syst Rehabil Eng. Vol.16, No.5 (2008), p.485-496.

[8] Yair Barniv, Mario Auilar, and Erion Hasnabelliu. Using EMG to Anticipate Head Motion for Virtual-Environment Applications. IEEE Trans. On Biomedical Engineering, Vol.52, No.6 (2005), p.1078-1093.

[9] P. O. Gutman and M. Velger, Tracking Tragets Using Adaptive Kalman Filtering, IEEE Trans. Aeorsp. Electron. Syst., Vol.26 (1988), p.691-698.

[10] Sun Zijun, Wu Dongsu, Shao Zheng. Analysis of Neck Muscles Related to Head Motion sEMG Acquisition for Virtual Cockpit Simulation. Aeronautical Computing Technique, Vol.44, No.1, (2014), p.115-122. 